\title{
Article \\ Oscillation and Nonoscillatory Criteria of Higher Order Dynamic Equations on Time Scales
}

\author{
Ya-Ru Zhu ${ }^{1}$, Zhong-Xuan Mao ${ }^{1, *}{ }^{\circledR}$, Jing-Feng Tian ${ }^{1}{ }^{1}$, Ya-Gang Zhang ${ }^{1}$ and Xin-Ni Lin ${ }^{2}$ \\ 1 Department of Mathematics and Physics, North China Electric Power University, Beijing 102206, China; \\ zhuyaru1982@ncepu.edu.cn (Y.-R.Z.); tianjf@ncepu.edu.cn (J.-F.T.); yagangzhang@ncepu.edu.cn (Y.-G.Z.) \\ 2 College of Foreign Languages, Cultures and International Exchanges, Zhejiang University, \\ Ningbo 315100, China; llxxnn@zju.edu.cn \\ * Correspondence: maozhongxuan@ncepu.edu.cn
}

check for updates

Citation: Zhu, Y.-R.; Mao, Z.-X.; Tian, J.-F.; Zhang, Y.-G.; Lin, X.-N. Oscillation and Nonoscillatory Criteria of Higher Order Dynamic Equations on Time Scales. Mathematics 2022, 10, 717. https:// doi.org/10.3390/math10050717

Academic Editor: Blanka Baculíková

Received: 24 January 2022

Accepted: 18 February 2022

Published: 24 February 2022

Publisher's Note: MDPI stays neutral with regard to jurisdictional claims in published maps and institutional affiliations.

Copyright: (C) 2022 by the authors. Licensee MDPI, Basel, Switzerland. This article is an open access article distributed under the terms and conditions of the Creative Commons Attribution (CC BY) license (https:// creativecommons.org/licenses/by/ $4.0 /)$.
Abstract: In this paper, we consider two universal higher order dynamic equations with several delay functions. We will establish two oscillatory criteria of the first equation and a sufficient and necessary condition for the second equation with a nonoscillatory solution by employing fixed point theorem.

Keywords: higher order dynamic equations; oscillation; nonoscillation; Riccati technique; fixed point theorem

\section{Introduction}

In 1988, Hilger [1] established a completely new theory in academia called time scales, which unites discrete and continuous representations and drew a lot of attention. The field of time scales has a tremendous amount of theoretical value, such as differential equations and difference equations can both be consolidated within the theoretical framework of dynamics equations on time scales. In this manner, it can avoid repeating studies in difference equations and differential equations while also exploring more similarities and differences between them. We refer the readers to [2-5] for more information about dynamic equations on time scales. Meanwhile, the study of dynamic equations had a wide range of applications in physics [6,7], chemistry [6], biology [6,8], engineering [6] and finance [9].

As we all know, most differential equations and difference equations with nonconstant coefficients, especially some nonlinear equations, have no analytical solution. Some researchers focus on the oscillatory and asymptotic behavior of the solutions to these equations, where a solution is oscillatory if it is neither finally positive nor eventually negative, and it is nonoscillatory if it is not oscillatory.

Since generic dynamic equations on time scales cannot be investigated directly due to technological constraints, researchers [10-25] usually confine their research to dynamic equations with specific structures under specified assumptions. Next, we list some equations that have been studied by scholars. However, it is worth pointing out that even while studying the same equation, various conditions can generate different results.

The authors $[10-19,23,24]$ mainly focused on the second order dynamic equation on time scales. Furthermore, the paper [15] provided by Agwo and Khodier in 2017 gave an uniform form of the above equations as follows:

$$
\left(a(t) g\left(x^{\Delta}(t)\right)\right)^{\Delta}+p(t) f(y(\tau(t)))=0, \quad t \in\left[t_{0}, \infty\right)_{\mathbb{T}} .
$$

As research advances, more attention is being paid to the oscillatory and asymptotic behaviour of higher order dynamic nonlinear equations [26-28]. For example, in 2012, Sun, $\mathrm{Yu}$ and $\mathrm{Xi}$ [26] discussed the oscillatory and asymptotic behavior of the following higher order dynamic equation: 


$$
\left(a_{n}(t)\left(\left(a_{n-1}(t)\left(\cdots\left(a_{1}(t) x^{\Delta}(t)\right)^{\Delta} \cdots\right)^{\Delta}\right)^{\Delta}\right)^{\gamma}\right)^{\Delta}+\delta p(t) f(x(\tau(t)))=0, \quad t \in\left[t_{0}, \infty\right)_{\mathbb{T}}
$$
criteria.

The two papers $[27,28]$ concerned the same equation and proposed different oscillatory

$$
\left(a_{n}(t)\left(\left(a_{n-1}(t)\left(\cdots\left(a_{1}(t) x^{\Delta}(t)\right)^{\Delta} \cdots\right)^{\Delta}\right)^{\Delta}\right)^{\gamma}\right)^{\Delta}+f(t, x(\tau(t)))=0, \quad t \in\left[t_{0}, \infty\right)_{\mathbb{T}} .
$$

Very recently, Chatzarakis, Grace and Jadlovská [25] provided a sharp oscillation for the following half-linear second-order differential equations with several delay terms:

$$
\left(a(t)\left|x^{\prime}(t)\right|^{\alpha-1} x^{\prime}(t)\right)^{\prime}+\sum_{i=1}^{m} q_{i}(t)\left|x^{\prime}\left(\tau_{i}(t)\right)\right|^{\alpha-1} x^{\prime}\left(\tau_{i}(t)\right)=0, \quad t \geq t_{0}>0 .
$$

On the contrary, scholars also concerned the nonoscillatory behavior of higher order dynamic equations [22,29-33]. For example, in 2009, Zhang, Dong and Li et al. [29] presented some sufficient conditions for the existence of positive solutions for

$$
(x(t)+p(t) x(\tau(t)))^{\Delta^{n}}+\Phi\left(t, x\left(\tau_{1}(t)\right), x\left(\tau_{2}(t)\right), \cdots, x\left(\tau_{m}(t)\right)\right)=0, \quad t \in\left[t_{0}, \infty\right)_{\mathbb{T}} .
$$

In 2010, some necessary and sufficient conditions for the following equation [30] were provided:

$$
\left(r(t)\left((x(t)-p(t) x(\delta(t)))^{\Delta^{m}}\right)^{\gamma}\right)^{\Delta}+\Phi(t, x(\tau(t)))=0, \quad t \in\left[t_{0}, \infty\right)_{\mathbb{T}} .
$$

Zhou [22] considered the following equation:

$$
(x(t)+p(t) x(t-\delta))^{\Delta^{n}}+\Phi(t, x(\tau(t)))=\alpha(t), \quad t \in\left[t_{0}, \infty\right)_{\mathbb{T}}
$$

Inspired by these works, we will establish oscillatory criteria for

$$
\begin{aligned}
& \left(\lambda_{n}(s) \Psi_{n}\left(\lambda_{n-1}(s) \Psi_{n-1}\left(\cdots\left(\lambda_{1}(s) \Psi_{1}\left(y^{\Delta}(s)\right)\right)^{\Delta} \cdots\right)^{\Delta}\right)^{\Delta}\right)^{\Delta} \\
& +\sum_{k=1}^{m} \phi_{k}(s) \varphi_{k}\left(y\left(\tau_{k}(s)\right)\right)=0, \quad s \in\left[s_{0}, \infty\right)_{\mathbb{T}} .
\end{aligned}
$$

and nonoscillatory criteria for

$$
\begin{aligned}
& \left(\lambda_{n}(s) \Psi_{n}\left(\lambda_{n-1}(s) \Psi_{n-1}\left(\cdots\left(\lambda_{1}(s) \Psi_{1}\left((y(s)+p(s) y(d(s)))^{\Delta}\right)\right)^{\Delta} \cdots\right)^{\Delta}\right)^{\Delta}\right)^{\Delta} \\
+ & \Phi\left(s, y\left(\tau_{1}(s)\right), y\left(\tau_{2}(s)\right), \cdots, y\left(\tau_{m}(s)\right)\right)=0, \quad s \in\left[s_{0}, \infty\right) \mathbb{T} .
\end{aligned}
$$

Comparing the previous equations with (8) and (9) yields that many existing research equations are covered by our research objects. Specifically, Equation (8) can be transformed into (2) by setting

$$
\Psi_{n}(s)=s^{\gamma}, \quad \Psi_{k}(s)=s,(k=1,2, \cdots, n-1), \quad m=1 .
$$

Equations (1) and (4) are special cases of (8) when $n=1, m=1$ and $n=1, m=1$, $\Psi_{1}(s)=\varphi_{1}(s)=|s|^{\alpha-1} s, \mathbb{T}=\mathbb{R}$, respectively. In the same way, it is easy to check that Equation (9) is a generalized form of Equations (5)-(7). It is also worth noting that Equation (9) can change into (8) by taking $p(s) \equiv 0$ and

$$
\Phi\left(s, y\left(\tau_{1}(s)\right), y\left(\tau_{2}(s)\right), \cdots, y\left(\tau_{m}(s)\right)\right)=\sum_{k=1}^{m} \phi_{k}(s) \varphi_{k}\left(y\left(\tau_{k}(s)\right)\right) .
$$


That is, we investigate equations in a broader form in this paper, which means that the oscillatory behavior of some equations that cannot be judged by current oscillatory criteria may be solved, such as, Examples 1 and 2 in Section 4.

This paper is organized as follows: Some notations and hypotheses are given in Section 4. In Section 3.1, we first provided some lemmas, and then two oscillatory criteria of the Equation (8) are established via lemmas. In Section 3.2, some useful lemmas are proposed, and we will constitute sufficient and necessary conditions for the existence of positive solutions to Equation (9). Some examples are furnished to show our results in Section 4. In the end, we summarize this paper in Section 5.

\section{Notations and Hypotheses}

For convenience, notations and hypotheses are listed in this section. First, using recursive definition, we rewrite Equation (8) as follows

$$
L_{n}^{\Delta}(s)+\sum_{k=1}^{m} \phi_{k}(s) \varphi_{k}\left(y\left(\tau_{k}(s)\right)\right)=0
$$

where $L_{k}(s)(k=0,1,2, \cdots, n)$ is defined as

$$
L_{k}(s)= \begin{cases}y(s), & \text { if } k=0, \\ \lambda_{k}(s) \Psi_{k}\left(L_{k-1}^{\Delta}(s)\right), & \text { if } k=1,2, \cdots, n .\end{cases}
$$

Likewise, Equation (9) can be rewritten as

$$
Q_{n}^{\Delta}(s)+\Phi\left(s, y\left(\tau_{1}(s)\right), y\left(\tau_{2}(s)\right), \cdots, y\left(\tau_{m}(s)\right)\right)=0,
$$

where $Q_{k}(s)(k=0,1,2, \cdots, n)$ is defined as

$$
Q_{k}(s)= \begin{cases}y(s)+p(s) y(d(s)), & \text { if } k=0, \\ \lambda_{k}(s) \Psi_{k}\left(Q_{k-1}^{\Delta}(s)\right), & \text { if } k=1,2, \cdots, n .\end{cases}
$$

For convenience, we denote

$$
\begin{aligned}
& T\{\Phi\}(s)=\int_{s}^{\infty} \Psi_{1}^{-1}\left(\frac { 1 } { \lambda _ { 1 } ( s _ { 1 } ) } \int _ { s _ { 1 } } ^ { \infty } \Psi _ { 2 } ^ { - 1 } \left(\frac { 1 } { \lambda _ { 2 } ( s _ { 2 } ) } \cdots \int _ { s _ { n - 1 } } ^ { \infty } \Psi _ { n } ^ { - 1 } \left(\frac{1}{\lambda_{n}\left(s_{n}\right)}\right.\right.\right. \\
& \left.\left.\int_{s_{n}}^{\infty} \Phi\left(s_{n+1}, y\left(\tau_{1}\left(s_{n+1}\right)\right), y\left(\tau_{2}\left(s_{n+1}\right)\right), \cdots, y\left(\tau_{m}\left(s_{n+1}\right)\right) \Delta s_{n+1}\right) \Delta s_{n} \cdots\right) \Delta s_{2}\right) \Delta s_{1},
\end{aligned}
$$

and

$$
\begin{aligned}
T^{*}\{\Phi(r)\}(s)= & \int_{s}^{\infty} \Psi_{1}^{-1}\left(\frac { 1 } { \lambda _ { 1 } ( s _ { 1 } ) } \int _ { s _ { 1 } } ^ { \infty } \Psi _ { 2 } ^ { - 1 } \left(\frac{1}{\lambda_{2}\left(s_{2}\right)} \cdots \int_{s_{n-1}}^{\infty} \Psi_{n}^{-1}\right.\right. \\
& \left.\left.\left(\frac{1}{\lambda_{n}\left(s_{n}\right)} \int_{s_{n}}^{\infty} \Phi\left(s_{n+1}, r, r, \cdots, r\right) \Delta s_{n+1}\right) \Delta s_{n} \cdots\right) \Delta s_{2}\right) \Delta s_{1},
\end{aligned}
$$

where $r>0$ is a constant.

We state the hypotheses in the following and will not repeat in the next section.

Hypothesis 1. $\mathbb{T}$ is an unbounded time scale, we write $\left[s_{0}, \infty\right) \cap \mathbb{T}$ as $\left[s_{0}, \infty\right)_{\mathbb{T}}$ and obediently assume $s_{0} \in \mathbb{T}$.

Hypothesis 2. Function $\Psi_{k}$ are odd, continuous and increasing, have inverse functions $\Psi_{k}^{-1}$ for all $k=1,2, \cdots, n$, and satisfy

$$
s \Psi_{k}(s)>0, \quad \Psi_{k}^{-1}(x y) \geq K \Psi_{k}^{-1}(x) \Psi_{k}^{-1}(y), \quad x, y>0, K>0, k=1,2, \cdots, n .
$$


Hypothesis 3. Function $\lambda_{k},(k=1,2, \cdots, n)$ are positive functions and satisfy

$$
\int_{s_{0}}^{\infty} \Psi_{k}^{-1}\left(\frac{1}{\lambda_{k}(s)}\right) \Delta s=\infty, \quad k=1,2, \cdots, n .
$$

Hypothesis 4. $\phi_{k}, \tau_{k}, \varphi_{k},(k=1,2, \cdots, n)$ are increasing continuous functions with $\lim _{s \rightarrow \infty} \tau_{k}(s)=\infty$. $\phi_{k}$ are positive functions and satisfy $s \phi_{k}^{\Delta}(s) \geq L \phi_{k}(s) . \varphi_{k}(s)$ satisfy $s \varphi_{k}(s)>0, \varphi_{k}(s) \geq s$.

\section{Hypothesis 5.}

$$
\int_{v}^{\infty} \Psi_{n-1}^{-1}\left(\frac{1}{\lambda_{n-1}(t)} \int_{t}^{\infty} \Psi_{n}^{-1}\left(\frac{1}{\lambda_{n}(s)} \int_{s}^{\infty} \sum_{k=1}^{m} \phi_{k}(u) \Delta u\right) \Delta s\right) \Delta t=\infty
$$

Hypothesis 6. Exists an $N_{1}>0$ subject to

$$
\frac{\varphi_{k}(t)}{\Psi_{n}\left(\Psi_{n-1}\left(\cdots \Psi_{1}(t)\right)\right)} \geq N_{1} \text { and } \tau_{k}(t) \geq t,
$$

for all $t \in\left[s_{0}, \infty\right)$ and $k=1,2, \cdots, n$.

Hypothesis 7. Exists an $\mathrm{N}_{2}>0$ subject to

$$
\frac{t \frac{\partial}{\Delta t} \Psi_{n}\left(\Psi_{n-1}\left(\cdots \Psi_{1}(t)\right)\right)}{\Psi_{n}\left(\Psi_{n-1}\left(\cdots \Psi_{1}(t)\right)\right)} \geq N_{2} \text { and } \Psi_{n}\left(\Psi_{n-1}\left(\cdots \Psi_{1}(t)\right)\right) \leq t,
$$

for all $t \in\left[s_{0}, \infty\right)$

Hypothesis 8. Function $p \not \equiv-1,1$. Function $\Phi\left(v_{0}, v_{1}, v_{2}, \cdots, v_{m}\right)$ is positive and increasing with respect to $v_{j}(j=0,1,2, \cdots, m)$. Exists a positive function $h(k)$ such that

$$
\Phi\left(v_{0}, k v_{1}, k v_{2}, \cdots, k v_{m}\right) \geq h(k) \Phi\left(v_{0}, v_{1}, v_{2}, \cdots, v_{m}\right) .
$$

\section{Hypothesis 9.}

$$
\Psi_{k}^{-1}(x y) \leq K_{2} \Psi_{k}^{-1}(x) \Psi_{k}^{-1}(y), \quad x, y>0, K_{2}>0, k=1,2, \cdots, n .
$$

\section{Main Results}

In what follows, we use (10) and (11) to refer to the equations we considered rather than (8) and (9).

\subsection{Oscillatory Criteria}

Before we establish oscillatory criteria in Theorems 1 and 2, we need the following four lemmas to explore some properties of Equation (10).

Lemma 1. Assume Hypotheses 1-3 hold, then the following conclusions are true.

(1) If $L_{p}(s)>0$ on $[S, \infty)$ and $\lim _{s \rightarrow \infty} L_{p}(s) \neq 0$, then $\lim _{s \rightarrow \infty} L_{j}(s)=\infty$ for all $j=$ $0,1,2, \cdots, p-1$.

(2) If $L_{p}(s)<0$ on $[S, \infty)$ and $\lim _{s \rightarrow \infty} L_{p}(s) \neq 0$, then $\lim _{s \rightarrow \infty} L_{j}(s)=-\infty$ for all $j=$ $0,1,2, \cdots, p-1$.

Proof. (1) Clearly, exists a $l_{1}>0$ such that $L_{p}(s) \geq l_{1}$ on $[S, \infty)$, that is,

$$
L_{p-1}^{\Delta}(s) \geq \Psi_{p}^{-1}\left(\frac{l_{1}}{\lambda_{p}(s)}\right) .
$$


Integrating from $S$ to $s$, we obtain

$$
L_{p-1}(s)-L_{p-1}(S) \geq \int_{S}^{s} \Psi_{p}^{-1}\left(\frac{l_{1}}{\lambda_{p}(s)}\right) \Delta s \geq K \Psi_{p}^{-1}\left(l_{1}\right) \int_{S}^{s} \Psi_{p}^{-1}\left(\frac{1}{\lambda_{p}(s)}\right) \Delta s,
$$

therefore, $\lim _{s \rightarrow \infty} L_{p-1}(s)=\infty$. Moreover, we have $\lim _{s \rightarrow \infty} L_{k}(s)=\infty(k=0,1, \cdots, p-2)$.

(2) In the same way, there exists a $l_{2}>0$ such that $L_{p}(s) \leq-l_{2}$ on $[S, \infty)$, noting that $\Psi_{k}$ are odd functions for all $k=1,2, \cdots, n$, we have

$$
L_{p-1}^{\Delta}(s) \leq \Psi_{p}^{-1}\left(\frac{-l_{2}}{\lambda_{p}(s)}\right)=-\Psi_{p}^{-1}\left(\frac{l_{2}}{\lambda_{p}(s)}\right) .
$$

Integrating from $S$ to $s$, we obtain

$$
L_{p-1}(s)-L_{p-1}(S) \leq-\int_{S}^{s} \Psi_{p}^{-1}\left(\frac{l_{2}}{\lambda_{p}(s)}\right) \Delta s \leq-K \Psi_{p}^{-1}\left(l_{2}\right) \int_{S}^{s} \Psi_{p}^{-1}\left(\frac{1}{\lambda_{p}(s)}\right) \Delta s,
$$

therefore, $\lim _{s \rightarrow \infty} L_{k}(s)=-\infty(k=0,1,2, \cdots, p-1)$.

Under certain conditions, the following lemma says there are only two possibilities if Equation (10) has an eventually positive solution.

Lemma 2. Assume Hypotheses 1-5 hold, Equation (10) has an eventually positive solution. Then one of the following conclusions holds.

(1) $L_{k}(s)>0$ for all $s>S$ and $k=0,1,2, \cdots, n$, where $S$ is a sufficiently large number.

(2) $\lim _{s \rightarrow \infty} y(s)=0$.

Proof. Based on the fact that Equation (10) have a eventually positive solution, therefore exits $s_{1}$ subject to $\min \left\{\tau_{1}\left(s_{1}\right), \tau_{2}\left(s_{1}\right), \cdots, \tau_{m}\left(s_{1}\right)\right\}>0$. Then

$$
\left(\lambda_{n}(s) \Psi_{n}\left(L_{n-1}^{\Delta}(s)\right)\right)^{\Delta}=-\sum_{k=1}^{m} \phi_{k}(s) \varphi_{k}\left(y\left(\tau_{k}(s)\right)\right)<0,
$$

which means $L_{n}=\lambda_{n} \Psi_{n}\left(L_{n-1}^{\Delta}\right)$ is strictly decreasing on $\left[s_{1}, \infty\right)$.

We claim that $L_{n}(s)>0$ on $\left[s_{1}, \infty\right)$, if not, there exists a $s_{2}$ such that $L_{n}(s)<0$ on $\left[s_{2}, \infty\right)$. Due to $L_{n}$ is strictly decreasing and $L_{n}(s)<0$, so $\lim _{s \rightarrow \infty} L_{n}(s)=0$ is impossible. Based on Lemma 1, we have $\lim _{s \rightarrow \infty} L_{j}(s)=-\infty$ for $j=0,1,2, \cdots, n-1$, which contradicts the fact that $y(s)$ is eventually positive.

Therefore, we know

$$
L_{n-1}^{\Delta}(s)=\Psi_{n}^{-1}\left(\frac{L_{n}(s)}{\lambda_{n}(s)}\right)>0,
$$

namely, $L_{n-1}$ is strictly increasing.

In the same way, if $L_{n-1}$ is eventually positive, then we have $\lim _{s \rightarrow \infty} L_{k}(s)=\infty,(k=$ $1,2, \cdots, n-2)$ by employing Lemma 1. Thus conclusion (1) holds. On the other hand, if $L_{n-1}<0$ for all $s \in\left[s_{1}, \infty\right)$, then

$$
L_{n-2}^{\Delta}=\Psi_{n-2}^{-1}\left(\frac{L_{n-1}(s)}{\lambda_{n-2}(s)}\right)<0,
$$

which means $L_{n-2}$ is strictly decreasing. Same as the cases $L_{n}$, we can deduce that $L_{n-2}(s)>$ 0 on $\left[s_{1}, \infty\right)$. We claim that the conclusion (2) hold. If not, we have $\lim _{s \rightarrow \infty} y(s)>0$, there exists $s_{3}$ and $M>0$ such that $\min \left\{y\left(\tau_{1}(s)\right), y\left(\tau_{2}(s)\right), \cdots, y\left(\tau_{m}(s)\right)\right\} \geq M$ on $\left[s_{3}, \infty\right)$. Integrating Equation (10) from $s$ to $\infty$, with respect to $s$, we can obtain

$$
\begin{aligned}
\lim _{s \rightarrow \infty} L_{n}(s)-L_{n}(s) & =-\int_{s}^{\infty} \sum_{k=1}^{m} \phi_{k}(u) \varphi_{k}\left(y\left(\tau_{k}(u)\right)\right) \Delta u \\
& \leq-\sum_{k=1}^{m} \varphi_{k}(M) \int_{s}^{\infty} \sum_{k=1}^{m} \phi_{k}(u) \Delta u,
\end{aligned}
$$


namely

$$
-L_{n}(s)=-\left(\lambda_{n}(s) \Psi_{n}\left(L_{n-1}^{\Delta}(s)\right) \leq-\sum_{k=1}^{m} \varphi_{k}(M) \int_{s}^{\infty} \sum_{k=1}^{m} \phi_{k}(u) \Delta u .\right.
$$

Integrating on both sides and using Hypothesis 2, we have

$$
\begin{aligned}
L_{n-1}(t) & \leq-\int_{t}^{\infty} \Psi_{n}^{-1}\left(\frac{\sum_{k=1}^{m} \varphi_{k}(M)}{\lambda_{n}(s)} \int_{s}^{\infty} \sum_{k=1}^{m} \phi_{k}(u) \Delta u\right) \Delta s \\
& \leq-K \Psi_{n}^{-1}\left(\sum_{k=1}^{m} \varphi_{k}(M)\right) \int_{t}^{\infty} \Psi_{n}^{-1}\left(\frac{1}{\lambda_{n}(s)} \int_{s}^{\infty} \sum_{k=1}^{m} \phi_{k}(u) \Delta u\right) \Delta s .
\end{aligned}
$$

Integrating and using Hypothesis 2 twice, we obtain

$$
-L_{n-2}(v) \leq-l_{2} \int_{v}^{\infty} \Psi_{n-1}^{-1}\left(\frac{1}{\lambda_{n-1}(t)} \int_{t}^{\infty} \Psi_{n}^{-1}\left(\frac{1}{\lambda_{n}(s)} \int_{s}^{\infty} \sum_{k=1}^{m} \phi_{k}(u) \Delta u\right) \Delta s\right) \Delta t,
$$

where $l_{2}=K^{2} \Psi_{n-1}^{-1}(K) \Psi_{n-1}^{-1}\left(\Psi_{n}^{-1}\left(\sum_{k=1}^{m} \varphi_{k}(M)\right)\right)>0$. We can derive the contradiction from Hypothesis 5 .

Lemma 3 establishes estimations of $L_{k}(s)$ and $L_{k}^{\Delta}(s),(k=0,1,2, \cdots, n-1)$ under the assumption that $\lim _{s \rightarrow \infty} y(s) \neq 0$, that is, we find function $f\left(s, L_{n}(s)\right)$ and $g\left(s, L_{n}(s)\right)$ such that $L_{k}(s) \geq f\left(s, L_{n}(s)\right), L_{k}(s) \geq g\left(s, L_{n}(s)\right)$ for all $s \in\left[s_{0}, \infty\right)$ and $k=0,1,2, \cdots, n-1$.

Lemma 3. Assume Hypotheses 1-3 and the case (1) in Lemma 2 hold, then

$$
L_{k}(s) \geq \eta_{k+1}(s) \Psi_{k+1}^{-1}\left(\Psi_{k+2}^{-1}\left(\cdots \Psi_{n}^{-1}\left(L_{n}(s)\right)\right)\right), \quad k=0,1,2, \cdots, n-1,
$$

and

$$
\begin{aligned}
& L_{k}^{\Delta}(s) \geq K \Psi_{k+1}^{-1}\left(\frac{\eta_{k+2}(s)}{\lambda_{k+1}(s)}\right) \Psi_{k+1}^{-1}\left(\Psi_{k+2}^{-1}\left(\Psi_{k+3}^{-1}\left(\cdots \Psi_{n}^{-1}\left(L_{n}(s)\right)\right)\right)\right), \quad k=0,1,2, \cdots, n-1, \\
& \text { where } \eta_{k}, k=1,2, \cdots, n+1 \text { are defined as } \\
& \eta_{k}(s)=\left\{\begin{array}{lll}
1, & \text { if } k=n+1, \\
K \int_{s_{1}}^{s} \Psi_{k}^{-1}\left(\frac{\eta_{k+1}(u)}{\lambda_{k}(u)}\right) \Delta u, & \text { if } k=1,2, \cdots, n .
\end{array}\right.
\end{aligned}
$$

Proof. Integrating $L_{n-1}^{\Delta}(s)=\Psi_{n}^{-1}\left(\frac{L_{n}(s)}{\lambda_{n}(s)}\right)$ on both sides from $s_{1}$ to $s$ and noting the fact that $L_{n}$ is strictly decreasing which we have proved in Lemma 2, we yield

$$
\begin{aligned}
L_{n-1}(s) & \geq \int_{s_{1}}^{s} \Psi_{n}^{-1}\left(\frac{L_{n}(u)}{\lambda_{n}(u)}\right) \Delta u \\
& \geq \int_{s_{1}}^{s} \Psi_{n}^{-1}\left(\frac{L_{n}(s)}{\lambda_{n}(u)}\right) \Delta u \\
& \geq K \Psi_{n}^{-1}\left(L_{n}(s)\right) \int_{s_{1}}^{s} \Psi_{n}^{-1}\left(\frac{1}{\lambda_{n}(u)}\right) \Delta u \\
& =\eta_{n}(s) \Psi_{n}^{-1}\left(L_{n}(s)\right) .
\end{aligned}
$$

Using inequality (16), we can immediately get 


$$
\begin{aligned}
L_{n-2}(s) & \geq \int_{s_{1}}^{s} \Psi_{n-1}^{-1}\left(\frac{L_{n-1}(u)}{\lambda_{n-1}(u)}\right) \Delta u \\
& \geq \int_{s_{1}}^{s} \Psi_{n-1}^{-1}\left(\frac{\eta_{n}(u) \Psi_{n}^{-1}\left(L_{n}(u)\right)}{\lambda_{n-1}(u)}\right) \Delta u \\
& \geq K \int_{s_{1}}^{s} \Psi_{n-1}^{-1}\left(\Psi_{n}^{-1}\left(L_{n}(u)\right)\right) \Psi_{n-1}^{-1}\left(\frac{\eta_{n}(u)}{\lambda_{n-1}(u)}\right) \Delta u \\
& \geq K \Psi_{n-1}^{-1}\left(\Psi_{n}^{-1}\left(L_{n}(s)\right)\right) \int_{s_{1}}^{s} \Psi_{n-1}^{-1}\left(\frac{\eta_{n}(u)}{\lambda_{n-1}(u)}\right) \Delta u \\
& =\eta_{n-1}(s) \Psi_{n-1}^{-1}\left(\Psi_{n}^{-1}\left(L_{n}(s)\right)\right) .
\end{aligned}
$$

We complete the proof by employing induction. Inequality (13) is held when $j=n$ and $j=n-1$. Supposing it's held for $j=k+1$, we will show the case $j=k$ is also true. More specifically, we know that

$$
L_{k+1}(s) \geq \eta_{k+2}(s) \Psi_{k+2}^{-1}\left(\Psi_{k+3}^{-1}\left(\ldots \Psi_{n}^{-1}\left(L_{n}(s)\right)\right)\right) .
$$

Consequently, integrating $L_{k}^{\Delta}(s)=\Psi_{k+1}\left(\frac{L_{k+1}(s)}{\lambda_{k+1}(s)}\right)$ from $s_{1}$ to $s$ on both side, we have

$$
\begin{aligned}
L_{k}(s) & \geq \int_{s_{1}}^{s} \Psi_{k+1}^{-1}\left(\frac{L_{k+1}(u)}{\lambda_{k+1}(u)}\right) \Delta u \\
& \geq \int_{s_{1}}^{s} K \Psi_{k+1}^{-1}\left(\Psi_{k+2}^{-1}\left(\Psi_{k+3}^{-1}\left(\ldots \Psi_{n}^{-1}\left(L_{n}(u)\right)\right)\right)\right) \Psi_{k+1}^{-1}\left(\frac{\eta_{k+2}(u)}{\lambda_{k+1}(u)}\right) \Delta u \\
& \geq \Psi_{k+1}^{-1}\left(\Psi_{k+2}^{-1}\left(\Psi_{k+3}^{-1}\left(\ldots \Psi_{n}^{-1}\left(L_{n}(s)\right)\right)\right)\right) \eta_{k+1}(s) .
\end{aligned}
$$

Hence, we can deduce the conclusion (13). Noting that $L_{k}^{\Delta}(s)=\Psi_{k+1}^{-1}\left(\frac{L_{k+1}(s)}{\lambda_{k+1}(s)}\right), k=$ $0,1,2, \cdots, n-1$, we have the following inequality by employing conclusion (13)

$$
\begin{aligned}
L_{k}^{\Delta}(s) & \geq \Psi_{k+1}^{-1}\left(\frac{\eta_{k+2}(s) \Psi_{k+2}^{-1}\left(\Psi_{k+3}^{-1}\left(\ldots \Psi_{n}^{-1}\left(L_{n}(s)\right)\right)\right)}{\lambda_{k+1}(s)}\right) \\
& \geq K \Psi_{k+1}^{-1}\left(\frac{\eta_{k+2}(s)}{\lambda_{k+1}(s)}\right) \Psi_{k+1}^{-1}\left(\Psi_{k+2}^{-1}\left(\Psi_{k+3}^{-1}\left(\cdots \Psi_{n}^{-1}\left(L_{n}(s)\right)\right)\right)\right),
\end{aligned}
$$

thereby, we complete the proof.

Lemma 4 is the chain rule on time scales and it will be used in Theorem 2.

Lemma 4 ([2], Theorem 2.57). Suppose function $f$ is continuous and function $g: \mathbb{T} \rightarrow \mathbb{R}$ is delta-differentiable. Then $f(g(x))$ is delta-differentiable with

$$
(f(g(x)))^{\Delta}=\left(\int_{0}^{1} f^{\Delta}\left(g(t)+h \mu(t) g^{\Delta}(t)\right) \mathrm{d} h\right) g^{\Delta}(t) .
$$

The following Theorem can be established only when it's based on Hypotheses 1-5 and Lemmas 1 and 2.

Theorem 1. Assume Hypotheses 1-5 hold and there exits a function $\delta(s)$ defined on $\mathbb{R}$ satisfies

$$
\int_{s_{1}}^{\infty} \delta(s) e_{-A(t)}\left(s, s_{1}\right) \Delta s=\infty
$$

where

$$
A(t)=\frac{\delta^{\Delta}(t)}{\delta^{\sigma}(t)}-\frac{L \delta(t)}{s \delta^{\sigma}(t)}
$$


Then Equation (10) is oscillatory or tends to zero.

Proof. We assume Equation (10) has an eventually positive or negative solution. Without loss of generality, we regard it positive. In fact, if $y(s)$ is an eventually negative solution, we can prove that $-y(s)$ is also a solution of Equation (10). If $y(s)$ tends to zero, then the proof is complete. Based on Lemma 2, we can assume $L_{k}(s)>0, k=0,1,2, \cdots, n$. Set

$$
\omega(s)=\delta(s)\left(\frac{L_{n}(s)}{\sum_{k=1}^{m} \phi_{k}(s) \varphi\left(y\left(\tau_{k}(s)\right)\right)}\right),
$$

$\omega(s)=\delta(s)\left(\frac{L_{n}(s)}{\Phi(s)}\right)$ for short, namely, we denote $\Phi(s)$ as $\sum_{k=1}^{m} \phi_{k}(s) \varphi\left(y\left(\tau_{k}(s)\right)\right)$. Differentiating $\omega$ with respect to $s$ and using the delta quotient rule, we have

$$
\begin{aligned}
\omega^{\Delta}(s) & =\left(\frac{\delta(s)}{\Phi(s)}\right) L_{n}^{\Delta}(s)+\left(\frac{\delta(s)}{\Phi(s)}\right)^{\Delta} L_{n}(\sigma(s)) \\
& =-\delta(s)+\left(\frac{\delta^{\Delta}(s) \Phi(s)-\delta(s) \Phi^{\Delta}(s)}{\Phi(s) \Phi^{\sigma}(s)}\right) L_{n}(\sigma(s)) \\
& =-\delta(s)+\frac{\omega^{\sigma}(s) \Phi^{\sigma}(s)}{\delta^{\sigma}(s)}\left(\frac{\delta^{\Delta}(s)}{\Phi^{\sigma}(s)}-\frac{\delta(s) \Phi^{\Delta}(s)}{\Phi(s) \Phi^{\sigma}(s)}\right) \\
& =-\delta(s)+\omega^{\sigma}(s) \frac{\delta^{\Delta}(s)}{\delta^{\sigma}(s)}-\omega^{\sigma}(s) \frac{\delta(s) \Phi^{\Delta}(s)}{\delta^{\sigma}(s) \Phi(s)} .
\end{aligned}
$$

Now using Hypothesis 4, we can obtain

$$
\begin{aligned}
\frac{\Phi^{\Delta}(s)}{\Phi(s)} & =\frac{\sum_{k=1}^{m}\left(\phi_{k}(s) \varphi\left(y\left(\tau_{k}(s)\right)\right)\right)^{\Delta}}{\sum_{k=1}^{m} \phi_{k}(s) \varphi\left(y\left(\tau_{k}(s)\right)\right)} \\
& =\frac{\sum_{k=1}^{m}\left(\phi_{k}^{\Delta}(s) \varphi\left(y\left(\tau_{k}(s)\right)\right)+\phi_{k}^{\sigma}(s) \varphi^{\Delta}\left(y\left(\tau_{k}(s)\right)\right)\right)}{\sum_{k=1}^{m} \phi_{k}(s) \varphi\left(y\left(\tau_{k}(s)\right)\right)} \\
& \geq \frac{L}{s} .
\end{aligned}
$$

Hence, we have

$$
\omega^{\Delta}(s) \leq-\delta(s)+\omega^{\sigma}(s) \frac{\delta^{\Delta}(s)}{\delta^{\sigma}(s)}-\omega^{\sigma}(s) \frac{L \delta(s)}{s \delta^{\sigma}(s)}=-\delta(s)+A(s) \omega^{\sigma}(s) .
$$

Note that

$$
\begin{aligned}
\left(\omega(s) e_{-A(s)}\left(s, s_{1}\right)\right)^{\Delta} & =\omega^{\Delta}(s) e_{-A(s)}\left(s, s_{1}\right)-A(s) \omega^{\sigma}(s) e_{-A(s)}\left(s, s_{1}\right) \\
& =\left(\omega^{\Delta}(s)-A(s) \omega^{\sigma}(s)\right) e_{-A(s)}\left(s, s_{1}\right) \leq-\delta(s) e_{-A(s)}\left(s, s_{1}\right),
\end{aligned}
$$

Delta integrating from $s_{1}$ to $s$ and letting $s \rightarrow \infty$, we have

$$
\lim _{s \rightarrow \infty} \omega(s) e_{-A(s)}\left(s, s_{1}\right)-\omega\left(s_{1}\right) e_{-A(s)}\left(s_{1}, s_{1}\right)+\lim _{s \rightarrow \infty} \int_{s_{1}}^{s} \delta(u) e_{-A(u)}\left(u, s_{1}\right) \Delta u \leq 0,
$$

which is a contradiction based on the condition (17), hence we complete the proof.

The second oscillatory criterion is established with more hypotheses and lemmas, however, it is more precise. In fact, this theorem has more applications for it has two arbitrary functions $\delta(s)$ and $m(s)$. 
Theorem 2. Assume Hypotheses 1-7 hold and there exit functions $\delta(s), m(s)$ defined on $\mathbb{R}$ satisfies

$$
\int_{s_{1}}^{\infty} B(s) e_{-\frac{\delta^{\Delta}(t)}{\delta^{\sigma}(t)}}\left(s, s_{1}\right) \Delta s=\infty,
$$

where

$$
\begin{aligned}
B(s) & =-N_{1} \delta(s) \sum_{k=1}^{m} \phi_{k}(s)+\delta(s)\left(\lambda_{n}(s) m(s)\right)^{\Delta} \\
& -\delta(s) \eta_{1}(s) K \Psi_{1}^{-1}\left(\frac{\eta_{2}(s)}{\lambda_{1}(s)}\right)\left(\int_{s}^{\infty} \sum_{k=1}^{m} \phi_{k}(t) \Delta t\right)^{2} \int_{\sigma(s)}^{\infty} \sum_{k=1}^{m} \phi_{k}(t) \Delta t .
\end{aligned}
$$

Then Equation (10) is oscillatory or tends to zero.

Proof. It suffices to prove Equation (10) is impossible to have an eventually positive solution is impossible under the assumption that $L_{k}(s)>0$ for all $k=0,1,2, \cdots, n$. We set

$$
\omega(s)=\delta(s)\left(\frac{L_{n}(s)}{P(y(s))}+\lambda_{n}(s) m(s)\right)
$$

where $P(v)=\Psi_{n}\left(\Psi_{n-1}\left(\cdots \Psi_{1}(v)\right)\right)$. Then

$$
\begin{aligned}
\omega^{\Delta}(s) & =\left(\delta(s) \frac{L_{n}(s)}{P(y(s))}\right)^{\Delta}+\left(\delta(s) \lambda_{n}(s) m(s)\right)^{\Delta} \\
& =\frac{\delta(s)}{P(y(s))} L_{n}^{\Delta}(s)+\left(\frac{\delta(s)}{P(y(s))}\right)^{\Delta} L_{n}^{\sigma}(s)+\delta(s)\left(\lambda_{n}(s) m(s)\right)^{\Delta}+\delta^{\Delta} \lambda_{n}^{\sigma}(s) m^{\sigma}(s) \\
& =\frac{\delta(s)}{P(y(s))} L_{n}^{\Delta}(s)+\left(\frac{\delta^{\Delta}(s)}{P^{\sigma}(y(s))}-\frac{\delta(s) P^{\Delta}(y(s))}{P(y(s)) P^{\sigma}(y(s))}\right) L_{n}^{\sigma}(s) \\
& +\delta(s)\left(\lambda_{n}(s) m(s)\right)^{\Delta}+\delta^{\Delta} \lambda_{n}^{\sigma}(s) m^{\sigma}(s) \\
& =-\frac{\delta(s)}{P(y(s))} \sum_{k=1}^{m} \phi_{k}(s) \varphi_{k}\left(y\left(\tau_{k}(s)\right)\right)+\left(\frac{\delta^{\Delta}(s)}{P^{\sigma}(y(s))}-\frac{\delta(s) P^{\Delta}(y(s))}{P(y(s)) P^{\sigma}(y(s))}\right) L_{n}^{\sigma}(s) \\
& +\delta(s)\left(\lambda_{n}(s) m(s)\right)^{\Delta}+\delta^{\Delta} \lambda_{n}^{\sigma}(s) m^{\sigma}(s) \\
& =-\frac{\delta(s)}{P(y(s))} \sum_{k=1}^{m} \phi_{k}(s) \varphi_{k}\left(y\left(\tau_{k}(s)\right)\right)+\delta(s)\left(\lambda_{n}(s) m(s)\right)^{\Delta}+\frac{\delta^{\Delta}(s)}{\delta^{\sigma}(s)} \omega^{\sigma}(s) \\
& -\delta(s) \frac{(P(y(s)))^{\Delta}}{P(y(s)) P^{\sigma}(y(s))} L_{n}^{\sigma}(s) .
\end{aligned}
$$

Based on Hypothesis 6, we have

$$
-\frac{\delta(s)}{P(y(s))} \sum_{k=1}^{m} \phi_{k}(s) \varphi_{k}\left(y\left(\tau_{k}(s)\right)\right) \leq-\frac{\delta(s)}{P(y(s))} \sum_{k=1}^{m} \phi_{k}(s) \varphi_{k}(y(s)) \leq-N \delta(s) \sum_{k=1}^{m} \phi_{k}(s) \text {. }
$$

Since $P(t) \leq t$ on $t \in\left[s_{0}, \infty\right)$, then $P^{\Delta \Delta} \leq 0$, i.e., $P^{\Delta}$ is decreasing. Then we have the following inequality by mean of Lemma 4 .

$$
\begin{aligned}
(P(y(s)))^{\Delta} & =\left(\int_{0}^{1} P^{\tilde{\Delta}}\left(h y^{\sigma}(s)+(1-h) y(s)\right) \mathrm{d} h\right) y^{\Delta}(s) \\
& \geq\left(\int_{0}^{1} P^{\tilde{\Delta}}\left(h y^{\sigma}(s)+(1-h) y^{\sigma}(s)\right) \mathrm{d} h\right) y^{\Delta}(s) \\
& =P^{\tilde{\Delta}}\left(y^{\sigma}(s)\right) y^{\Delta}(s)
\end{aligned}
$$


where $P^{\tilde{\Delta}}\left(y^{\sigma}(s)\right)$ means derivative $P$ with respect to $y$ rather than $s$. Noting that

$$
\begin{aligned}
y^{\Delta}(s) & =\frac{\left(\Psi_{1}^{-1}\left(\Psi_{2}^{-1}\left(\ldots \Psi_{n}^{-1}\left(L_{n}(s)\right)\right)\right)^{2}\right.}{y(s)} \frac{y(s)}{\Psi_{1}^{-1}\left(\Psi_{2}^{-1}\left(\cdots \Psi_{n}^{-1}\left(L_{n}(s)\right)\right)\right)} \frac{y^{\Delta}(s)}{\Psi_{1}^{-1}\left(\Psi_{2}^{-1}\left(\ldots \Psi_{n}^{-1}\left(L_{n}(s)\right)\right)\right)} \\
& \geq \frac{\left(\Psi_{1}^{-1}\left(\Psi_{2}^{-1}\left(\ldots \Psi_{n}^{-1}\left(L_{n}(s)\right)\right)\right)^{2}\right.}{y(s)} \eta_{1}(s) K \Psi_{1}^{-1}\left(\frac{\eta_{2}(s)}{\lambda_{1}(s)}\right),
\end{aligned}
$$

then inequality (19) leads to

$$
\begin{aligned}
\omega^{\Delta}(s) & =-\frac{\delta(s)}{P(y(s))} \sum_{k=1}^{m} \phi_{k}(s) \varphi_{k}\left(y\left(\tau_{k}(s)\right)\right)+\delta(s)\left(\lambda_{n}(s) m(s)\right)^{\Delta}+\frac{\delta^{\Delta}(s)}{\delta^{\sigma}(s)} \omega^{\sigma}(s) \\
& -\delta(s) \frac{(P(y(s)))^{\Delta}}{P(y(s)) P^{\sigma}(y(s))} L_{n}^{\sigma}(s) \\
\leq & -N \delta(s) \sum_{k=1}^{m} \phi_{k}(s)+\delta(s)\left(\lambda_{n}(s) m(s)\right)^{\Delta}+\frac{\delta^{\Delta}(s)}{\delta^{\sigma}(s)} \omega^{\sigma}(s) \\
& -\delta(s) \frac{P^{\tilde{\Delta}}\left(y^{\sigma}(s)\right) y^{\Delta}(s)}{P(y(s)) P^{\sigma}(y(s))} L_{n}^{\sigma}(s) \\
\leq & -N \delta(s) \sum_{k=1}^{m} \phi_{k}(s)+\delta(s)\left(\lambda_{n}(s) m(s)\right)^{\Delta}+\frac{\delta^{\Delta}(s)}{\delta^{\sigma}(s)} \omega^{\sigma}(s) \\
& -\delta(s) \frac{P^{\tilde{\Delta}}\left(y^{\sigma}(s)\right)}{P\left(y^{\sigma}(s)\right)} \frac{\left(\Psi_{1}^{-1}\left(\Psi_{2}^{-1}\left(\cdots \Psi_{n}^{-1}\left(L_{n}(s)\right)\right)\right)^{2}\right.}{y(s)} \eta_{1}(s) K \Psi_{1}^{-1}\left(\frac{\eta_{2}(s)}{\lambda_{1}(s)}\right) \frac{L_{n}^{\sigma}(s)}{P^{\sigma}(y(s))}
\end{aligned}
$$

By employing Hypothesis 7, we know $P(t) \leq t$ and $\Psi_{1}^{-1}\left(\Psi_{2}^{-1}\left(\ldots \Psi_{n}^{-1}(t)\right)\right) \geq t$, therefore

$$
\begin{aligned}
\omega^{\Delta}(s) & \leq-N \delta(s) \sum_{k=1}^{m} \phi_{k}(s)+\delta(s)\left(\lambda_{n}(s) m(s)\right)^{\Delta}+\frac{\delta^{\Delta}(s)}{\delta^{\sigma}(s)} \omega^{\sigma}(s) \\
& -\delta(s)\left(\frac{L_{n}(s)}{y(s)}\right)\left(\frac{L_{n}(s)}{y^{\sigma}(s)}\right) \eta_{1}(s) K \Psi_{1}^{-1}\left(\frac{\eta_{2}(s)}{\lambda_{1}(s)}\right)\left(\frac{L_{n}(s)}{y(s)}\right)^{\sigma} .
\end{aligned}
$$

Finally, we find a lower bound of $\frac{L_{n}(s)}{y(s)}$. In fact, integrating Equation (10), we have

$$
\begin{aligned}
L_{n}(s) & \geq \int_{s}^{\infty} \sum_{k=1}^{m} \phi_{k}(t) \varphi_{k}\left(y\left(\tau_{k}(t)\right)\right) \Delta t \\
& \geq \int_{s}^{\infty} \sum_{k=1}^{m} \phi_{k}(t) \varphi_{k}\left(y^{\sigma}(t)\right) \Delta t \\
& \geq y^{\sigma}(s) \int_{s}^{\infty} \sum_{k=1}^{m} \phi_{k}(t) \Delta t \\
& \geq y(s) \int_{s}^{\infty} \sum_{k=1}^{m} \phi_{k}(t) \Delta t .
\end{aligned}
$$

Therefore, we arrive at

$$
\begin{aligned}
\omega^{\Delta}(s) & \leq-N \delta(s) \sum_{k=1}^{m} \phi_{k}(s)+\delta(s)\left(\lambda_{n}(s) m(s)\right)^{\Delta}+\frac{\delta^{\Delta}(s)}{\delta^{\sigma}(s)} \omega^{\sigma}(s) \\
& -\delta(s) \eta_{1}(s) K \Psi_{1}^{-1}\left(\frac{\eta_{2}(s)}{\lambda_{1}(s)}\right)\left(\int_{s}^{\infty} \sum_{k=1}^{m} \phi_{k}(t) \Delta t\right)^{2} \int_{\sigma(s)}^{\infty} \sum_{k=1}^{m} \phi_{k}(t) \Delta t \\
& =\frac{\delta^{\Delta}(s)}{\delta^{\sigma}(s)} \omega^{\sigma}(s)+B(s) .
\end{aligned}
$$


Likewise, noting that

$$
\left(\omega(s) e_{-\frac{\delta^{\Delta}(t)}{\delta^{\sigma}(t)}}\left(s, s_{1}\right)\right)^{\Delta} \leq B(s) e_{-\frac{\delta^{\Delta}(t)}{\delta^{\sigma}(t)}}\left(s, s_{1}\right),
$$

Delta integrating from $s_{1}$ to $s$ and letting $s \rightarrow \infty$, we can deduce a contradiction based on the condition (18), hence we complete the proof.

\subsection{Nonoscillatory Criteria}

Same as Section 3.1, the following lemma which explores the properties of Equation (11) is given at the first place.

Lemma 5. If Equation (11) has a bounded positive solution and Hypotheses 1-3 and 8 hold, then we have the following conclusions.

(1) $(-1)^{n-j+1} Q_{j}^{\Delta}(s)>0$ for all $j=0,1,2, \cdots, n$.

(2) $\quad(-1)^{n-j} Q_{j}(s)>0$ for all $j=1,2, \cdots, n$.

(3) $\lim _{s \rightarrow \infty} Q_{j}(s)=0$ for all $j=1,2, \cdots, n$.

Proof. Based on Equation (11) and Hypothesis 8, we have

$$
Q_{n}^{\Delta}(s)=-\Phi\left(s, y\left(\tau_{1}(s)\right), y\left(\tau_{2}(s)\right), \cdots, y\left(\tau_{m}(s)\right)\right)<0 .
$$

We claim that $Q_{n}(s)>0$ and $\lim _{s \rightarrow \infty} Q_{n}(s)=0$. If not, there exits $s_{1}$ and $l_{1}>0$ such that $\left|Q_{n}(s)\right| \geq l_{1}$ on $\left[s_{1}, \infty\right)$. If $Q_{n}(s)$ is eventually positive, then

$$
Q_{n-1}(s)-Q_{n-1}\left(s_{1}\right) \geq \int_{s_{1}}^{s} \Psi_{n}^{-1}\left(\frac{l_{1}}{\lambda_{n}(s)}\right) \Delta s \geq K \Psi_{n}^{-1}\left(l_{1}\right) \int_{s_{1}}^{s} \Psi_{n}^{-1}\left(\frac{1}{\lambda_{n}(s)}\right) \Delta s,
$$

therefore $\lim _{s \rightarrow \infty} Q_{k}(s)=\infty(k=0,1, \cdots, n-1)$, which contradicts the fact that $Q_{0}(s)=$ $y(s)+p(s) y(d(s))$ is bounded. If $Q_{n}(s)$ is eventually negative, it can deduce $\lim _{s \rightarrow \infty} Q_{k}(s)=$ $-\infty(k=0,1, \cdots, n-1)$ in the same way. It also contradicts the conclusion that $Q_{0}(s)$ is bounded.

Noting that $Q_{n-1}^{\Delta}=\Psi_{n}\left(\frac{Q_{n}(s)}{\lambda_{n}(s)}\right)>0$, We can know that $Q_{n-1}(s)<0$ and $\lim _{s \rightarrow \infty} Q_{n-1}(s)=0$. Repeating the process, we can get the conclusions.

Next two lemmas are about fixed point theorem which can be found in $[29,34]$.

Lemma 6 ([29]). If $f^{\Delta}$ is uniformly bounded, then $f$ is equicontinuous.

Lemma 7 (Kranoselskii's fixed point theorem, see [34]). If $\Omega \subset X$ is closed, convex and bounded, exist two maps $\mathcal{L}_{1}$ and $\mathcal{L}_{2}: \Omega \rightarrow X$ such that

(1) $\mathcal{L}_{1} y_{1}+\mathcal{L}_{2} y_{2} \in \Omega$ for all $y_{1}, y_{2} \in \Omega$,

(2) $\mathcal{L}_{1}$ is a contraction,

(3) $\mathcal{L}_{2}$ is completely continuous.

Then the equation

$$
\mathcal{L}_{1} y+\mathcal{L}_{2} y=y,
$$

has a solution in $\Omega$.

The following Theorem establishes a sufficient and necessary condition for the existence of positive solution for Equation (11).

Theorem 3. If Hypotheses 1-3, 8 and 9 hold, then Equation (11) has an eventually bounded nonoscillatory solution $y(s)$ with $\lim _{s \rightarrow \infty} y(s) \neq 0$ if and only if $T^{*}\{\Phi(u)\}(s)<\infty$, where $T^{*}\{\Phi(u)\}(s)$ is defined by (12) and $u \geq \max _{s \in\left[s_{0}, \infty\right)} y(s)$. 
Proof. Sufficiency. If Equation (11) has an eventually positive bounded solution, then there exist constant $w$ and $S$ such that $\min \left\{y(s), y\left(\tau_{1}(s)\right), y\left(\tau_{2}(s)\right), \cdots, y\left(\tau_{m}(s)\right)\right\}>u / w$ on $[S, \infty)$. Noting that

$$
\begin{array}{r}
Q_{n}^{\Delta}(s)=-\Phi\left(s, y\left(\tau_{1}(s)\right), y\left(\tau_{2}(s)\right), \cdots, y\left(\tau_{m}(s)\right)\right. \\
<-\Phi\left(s, \frac{u}{w}, \frac{u}{w}, \cdots, \frac{u}{w}\right) \leq-h\left(\frac{1}{w}\right) \Phi(s, u, u, \cdots, u),
\end{array}
$$

integrating from $s_{n}>S$ to $\infty$, we have

$$
Q_{n}\left(s_{n}\right)>h\left(\frac{1}{w}\right) \int_{s_{n}}^{\infty} \Phi\left(s_{n+1}, u, u, \cdots, u\right) \Delta s_{n+1} .
$$

Together with the conclusions (2) and (3) in Lemma 5, inequality (22) and Hypothesis 9, we have

$$
\begin{aligned}
& T^{*}\{\Phi(u)\}(s) \\
= & \int_{s}^{\infty} \Psi_{1}^{-1}\left(\frac { 1 } { \lambda _ { 1 } ( s _ { 1 } ) } \int _ { s _ { 1 } } ^ { \infty } \Psi _ { 2 } ^ { - 1 } \left(\frac{1}{\lambda_{2}\left(s_{2}\right)} \cdots \int_{s_{n-1}}^{\infty} \Psi_{n}^{-1}\right.\right. \\
& \left.\left.\left(\frac{1}{\lambda_{n}\left(s_{n}\right)} \int_{s_{n}}^{\infty} \Phi\left(s_{n+1}, u, u, \cdots, u\right) \Delta s_{n+1}\right) \Delta s_{n} \cdots\right) \Delta s_{2}\right) \Delta s_{1} \\
\leq & \int_{s}^{\infty} \Psi_{1}^{-1}\left(\frac{1}{\lambda_{1}\left(s_{1}\right)} \int_{s_{1}}^{\infty} \Psi_{2}^{-1}\left(\frac{1}{\lambda_{2}\left(s_{2}\right)} \cdots \int_{s_{n-1}}^{\infty} \Psi_{n}^{-1}\left(\frac{1}{\lambda_{n}\left(s_{n}\right) h\left(\frac{1}{w}\right)} Q_{n}\left(s_{n}\right)\right) \Delta s_{n} \cdots\right) \Delta s_{2}\right) \Delta s_{1} \\
\leq & \int_{s}^{\infty} \Psi_{1}^{-1}\left(\frac{1}{\lambda_{1}\left(s_{1}\right)} \int_{s_{1}}^{\infty} \Psi_{2}^{-1}\left(\frac{1}{\lambda_{2}\left(s_{2}\right)} \cdots \int_{s_{n-1}}^{\infty} K_{2} \Psi_{n}^{-1}\left(\frac{1}{h\left(\frac{1}{w}\right)}\right) Q_{n-1}^{\Delta}\left(s_{n}\right) \Delta s_{n} \cdots\right) \Delta s_{2}\right) \Delta s_{1} \\
= & \int_{s}^{\infty} \Psi_{1}^{-1}\left(\frac { 1 } { \lambda _ { 1 } ( s _ { 1 } ) } \int _ { s _ { 1 } } ^ { \infty } \Psi _ { 2 } ^ { - 1 } \left(\frac{1}{\lambda_{2}\left(s_{2}\right)} \cdots \int_{s_{n-2}}^{\infty} \Psi_{n-1}^{-1}\right.\right. \\
& \left.\left(K_{2} \Psi_{n}^{-1}\left(\frac{1}{h\left(\frac{1}{w}\right)}\right) \frac{1}{\lambda_{n-1}\left(s_{2}\right)}\left(\frac{\lim }{s_{n-1} \rightarrow \infty} Q_{n-1}\left(s_{n-1}\right)-Q_{n-1}\left(s_{n-1}\right)\right) \Delta s_{n-1} \cdots\right) \Delta s_{2}\right) \Delta s_{1} \\
= & \int_{s}^{\infty} \Psi_{1}^{-1}\left(\frac { 1 } { \lambda _ { 1 } ( s _ { 1 } ) } \int _ { s _ { 1 } } ^ { \infty } \Psi _ { 2 } ^ { - 1 } \left(\frac{1}{\lambda_{2}\left(s_{2}\right)} \cdots\right.\right. \\
& \left.\int_{s_{n-2}}^{\infty} \Psi_{n-1}^{-1}\left(K_{2} \Psi_{n}^{-1}\left(\frac{1}{h\left(\frac{1}{w}\right)}\right) \frac{1}{\lambda_{n-1}\left(s_{2}\right)}\left(-Q_{n-1}\left(s_{n-1}\right)\right) \Delta s_{n-1} \cdots\right) \Delta s_{2}\right) \Delta s_{1} \\
\leq & \int_{s}^{\infty} \Psi_{1}^{-1}\left(\frac { 1 } { \lambda _ { 1 } ( s _ { 1 } ) } \int _ { s _ { 1 } } ^ { \infty } \Psi _ { 2 } ^ { - 1 } \left(\frac{1}{\lambda_{2}\left(s_{2}\right)} \cdots\right.\right. \\
& \left.\left.K_{2} \Psi_{n-1}^{-1}\left(K \Psi_{n}^{-1}\left(\frac{1}{h\left(\frac{1}{w}\right)}\right)\right) \int_{s_{n-2}}^{\infty}-Q_{n-2}^{\Delta}\left(s_{n-1}\right) \Delta s_{n-1} \cdots\right) \Delta s_{2}\right) \Delta s_{1} \\
= & \int_{s}^{\infty} \Psi_{1}^{-1}\left(\frac { 1 } { \lambda _ { 1 } ( s _ { 1 } ) } \int _ { s _ { 1 } } ^ { \infty } \Psi _ { 2 } ^ { - 1 } \left(\frac{1}{\lambda_{2}\left(s_{2}\right)} \cdots\right.\right. \\
& \left.\left.\int_{s_{n-3}}^{\infty} \Psi_{n-2}^{-1}\left(K_{2} \Psi_{n-1}^{-1}\left(K_{2} \Psi_{n}^{-1}\left(\frac{1}{h\left(\frac{1}{w}\right)}\right)\right) \frac{1}{\lambda_{n-2}(s)} Q_{n-2}\left(s_{n-2}\right)\right) \Delta s_{n-2} \cdots\right) \Delta s_{2}\right) \Delta s_{1} \\
\leq & \cdots \\
\leq & K^{*}(-1)^{n} Q_{0}(s)<\infty,
\end{aligned}
$$

where

$$
k^{*}=K \Psi_{1}^{-1}\left(K_{2} \Psi_{2}^{-1}\left(K_{2} \Psi_{3}^{-1}\left(\cdots K_{2} \Psi_{n-1}^{-1}\left(K_{2} \Psi_{n}^{-1}\left(\frac{1}{h\left(\frac{1}{w}\right)}\right)\right) \cdots\right)\right)\right) .
$$

Thereby we complete the proof.

Necessity. Case 1: $-1<p_{1} \leq p(s) \leq 0$. Supposing solution $y(s)$ satisfies $l \leq y(s) \leq u$ on $\left[s_{1}, \infty\right)$, we define

$$
\Omega=\left\{y \in C_{r d}\left(\left[s_{0}, \infty\right),(-\infty, \infty)\right): l \leq y(s) \leq u, s \geq s_{0}\right\} .
$$


Clearly, $\Omega$ is a bounded, convex, and closed subset of $C_{r d}\left(\left[s_{0}, \infty\right),(-\infty, \infty)\right)$ which is a Banach space.

Since $T^{*}\{\Phi(u)\}(s)<\infty$, there exists $S>s_{0}$ such that

$$
T^{*}\{\Phi(u)\}(s) \leq \frac{\left(1+p_{1}\right)(u-l)}{2}, \quad s \in[S, \infty) .
$$

We set

$$
\left(\mathcal{L}_{1} y\right)(s)= \begin{cases}\frac{\left(1+p_{1}\right)(l+u)}{2}-p(s) y(d(s)), & s \geq S \\ \left(\mathcal{L}_{1} y\right)(S), & S \geq s \geq s_{0}\end{cases}
$$

and

$$
\left(\mathcal{L}_{2} y\right)(s)= \begin{cases}(-1)^{n} T\{\Phi\}(s), & s \geq s, \\ \left(\mathcal{L}_{2} y\right)(S), & S \geq s \geq s_{0} .\end{cases}
$$

1. We claim $\left(\mathcal{L}_{1} y_{1}\right)(s)+\left(\mathcal{L}_{2} y_{2}\right)(s) \in \Omega$ for all $y_{1}, y_{2} \in \Omega$. Clearly, we have

$$
\begin{aligned}
\left(\mathcal{L}_{1} y_{1}\right)(s)+\left(\mathcal{L}_{2} y_{2}\right)(s) & \leq \frac{\left(1+p_{1}\right)(l+u)}{2}-p_{1} u+T\{\Phi\}(s) \\
& \leq \frac{\left(1+p_{1}\right)(l+u)}{2}-p_{1} u+\int_{s}^{\infty} \Psi_{1}^{-1}\left(\frac { 1 } { \lambda _ { 1 } ( s _ { 1 } ) } \int _ { s _ { 1 } } ^ { \infty } \Psi _ { 2 } ^ { - 1 } \left(\frac{1}{\lambda_{2}\left(s_{2}\right)} \cdots\right.\right. \\
& \left.\left.\int_{s_{n-1}}^{\infty} \Psi_{n}^{-1}\left(\frac{1}{\lambda_{n}\left(s_{n}\right)} \int_{s_{n}}^{\infty} \Phi\left(s_{n+1}, u, u, \cdots, u\right) \Delta s_{n+1}\right) \Delta s_{n} \cdots\right) \Delta s_{2}\right) \Delta s_{1} \\
\leq & \frac{\left(1+p_{1}\right)(l+u)}{2}-p_{1} u+\frac{\left(1+p_{1}\right)(u-l)}{2}=u
\end{aligned}
$$

Moreover, the following inequalities hold

$$
\begin{aligned}
\left(\mathcal{L}_{1} y_{1}\right)(s)+\left(\mathcal{L}_{2} y_{2}\right)(s) & \geq \frac{\left(1+p_{1}\right)(l+u)}{2}-p_{1} l-T\{\Phi\}(s) \\
& \geq \frac{\left(1+p_{1}\right)(l+u)}{2}-p_{1} l-\frac{\left(1+p_{1}\right)(u-l)}{2}=l
\end{aligned}
$$

Hence, we have proved that $\left(\mathcal{L}_{1} y_{1}\right)(s)+\left(\mathcal{L}_{2} y_{2}\right)(s) \in \Omega$ for all $y_{1}, y_{2} \in \Omega$.

2. We claim $\left(\mathcal{L}_{1} y\right)(s)$ is a contraction on $\Omega$. It is clear that

$$
\left|\left(\mathcal{L}_{1} y_{1}\right)(s)-\left(\mathcal{L}_{2} y_{2}\right)(s)\right| \leq-p_{1}\left|y_{1}(d(s))-y_{2}(d(s))\right| \leq-p_{1}\left\|y_{1}-y_{2}\right\|, \quad s>S,
$$

where $\|x\|=\sup _{s>s_{0}}|x(s)|$. It completes the proof.

3. We claim $\left(\mathcal{L}_{2} y\right)(s)$ is completely continuous. We have that proved $\mathcal{L}_{2} \Omega$ is uniformly bounded. Note the following inequalities hold

$$
\begin{aligned}
& \left|\left(\mathcal{L}_{2} y\right)^{\Delta}(s)\right| \\
& =\mid \Psi_{1}^{-1}\left(\frac { 1 } { \lambda _ { 1 } ( s ) } \int _ { s } ^ { \infty } \Psi _ { 2 } ^ { - 1 } \left(\frac{1}{\lambda_{2}\left(s_{2}\right)} \cdots \int_{s_{n-1}}^{\infty} \Psi_{n}^{-1}\right.\right. \\
& \left.\left(\frac{1}{\lambda_{n}\left(s_{n}\right)} \int_{s_{n}}^{\infty} \Phi\left(s_{n+1}, y\left(\tau_{1}\left(s_{n+1}\right)\right), y\left(\tau_{2}\left(s_{n+1}\right)\right), \cdots, y\left(\tau_{m}\left(s_{n+1}\right)\right)\right) \Delta s_{n+1}\right) \Delta s_{n} \cdots\right) \Delta s_{2} \mid \\
& \leq \mid \Psi_{1}^{-1}\left(\frac{1}{\lambda_{1}(s)} \int_{s}^{\infty} \Psi_{2}^{-1}\left(\frac{1}{\lambda_{2}\left(s_{2}\right)} \cdots \int_{s_{n-1}}^{\infty} \Psi_{n}^{-1}\left(\frac{1}{\lambda_{n}\left(s_{n}\right)} \int_{s_{n}}^{\infty} \Phi\left(s_{n+1}, u, u, \cdots, u\right) \Delta s_{n+1}\right) \Delta s_{n} \cdots\right) \Delta s_{2} \mid .\right.
\end{aligned}
$$

Thus, we deduce that $\mathcal{L}_{2} \Omega$ is uniformly bounded based on Lemma 6 .

Now using Lemma 7, there exist a solution of equation $\mathcal{L}_{1} y+\mathcal{L}_{2} y=y$, which means Equation (11) has an eventually bounded positive solution. 
Case 2. If $-\infty<p(s) \leq p_{2}<-1$. Then we can deduce the same conclusion in Case 1 by setting

$$
\begin{gathered}
\left(\mathcal{L}_{1} y\right)(s)= \begin{cases}\frac{\left(1+p_{2}\right)(l+u)}{2 p_{2}}-\frac{1}{p\left(d^{-1}(s)\right)} y\left(d^{-1}(s)\right), & s \geq S, \\
\left(\mathcal{L}_{1} y\right)(S), & S \geq s \geq s_{0},\end{cases} \\
\left(\mathcal{L}_{2} y\right)(s)= \begin{cases}\frac{(-1)^{n+1}}{p\left(d^{-1}(s)\right)} T\{\Phi\}\left(d^{-1}(s)\right), & s \geq S, \\
\left(\mathcal{L}_{2} y\right)(S), & S \geq s \geq s_{0} .\end{cases}
\end{gathered}
$$

and $T^{*}\{\Phi(u)\}(s) \leq-\frac{\left(p_{2}+1\right)(l-u)}{2}$.

Case 3. If $0 \leq p(s) \leq p_{3}<1$. In the same way, we take

$$
\left(\mathcal{L}_{1} y\right)(s)= \begin{cases}\frac{\left(1+p_{3}\right)(l+u)}{2}-p(s) y(d(s)), & s \geq S \\ \left(\mathcal{L}_{1} y\right)(S), & S \geq s \geq s_{0}\end{cases}
$$

$\left(\mathcal{L}_{2} y\right)(s)$ same as case 1 and $T^{*}\{\Phi(u)\}(s) \leq \frac{\left(1-p_{3}\right)(l-u)}{2}$.

Case 4 . If $1<p_{4} \leq p(s)<\infty$. It is sufficient to let

$$
\left(\mathcal{L}_{1} y\right)(s)= \begin{cases}\frac{\left(1+p_{4}\right)(l+u)}{2 p_{4}}-\frac{1}{p\left(d^{-1}(s)\right)} y\left(d^{-1}(s)\right), & s \geq S \\ \left(\mathcal{L}_{1} y\right)(S), & S \geq s \geq s_{0}\end{cases}
$$

$\left(\mathcal{L}_{2} y\right)(s)$ same as case 2 and $T^{*}\{\Phi(u)\}(s) \leq \frac{\left(p_{4}-1\right)(l-u)}{2}$. Thereby we complete the proof.

If we take $p(s) \equiv 0$ and

$$
\Phi\left(s, y\left(\tau_{1}(s)\right), y\left(\tau_{2}(s)\right), \cdots, y\left(\tau_{m}(s)\right)\right)=\sum_{k=1}^{m} \phi_{k}(s) \varphi_{k}\left(y\left(\tau_{k}(s)\right)\right),
$$

then we have the following corollary.

Corollary 1. If Hypotheses 1-4 and 9 hold, then Equation (10) has an eventually bounded nonoscillatory solution $y(s)$ with $\lim _{s \rightarrow \infty} y(s) \neq 0$ if and only if there exits $u$ such that

$$
\int_{s}^{\infty} \Psi_{1}^{-1}\left(\frac{1}{\lambda_{1}\left(s_{1}\right)} \int_{s_{1}}^{\infty} \Psi_{2}^{-1}\left(\frac{1}{\lambda_{2}\left(s_{2}\right)} \cdots \int_{s_{n-1}}^{\infty} \Psi_{n}^{-1}\left(\frac{1}{\lambda_{n}\left(s_{n}\right)} \int_{s_{n}}^{\infty} \sum_{k=1}^{m} \phi_{k}\left(s_{n}\right) \varphi_{k}(u) \Delta s_{n+1}\right) \Delta s_{n} \cdots\right) \Delta s_{2}\right) \Delta s_{1},
$$

is finite, where $u$ is satisfying $u \geq \max _{s \in\left[s_{0}, \infty\right)} y(s)$.

\section{Examples}

Example 1. Consider the equation on $\mathbb{T}=\mathbb{N}$,

$$
L_{n}(s)+\sum_{k=1}^{m} s^{c_{1, k}}\left(y\left(\tau_{k}(s)\right)\right)^{c_{2, k}}=0, \quad s \in[2, \infty)_{\mathbb{N}}
$$

where $\Psi_{k}(s)=s^{\gamma_{k}}, \lambda_{k}(s)=s^{\beta_{k}}$ and $\gamma_{k}$ are the quotient of odd positive integers satisfies

$$
\beta_{k} \leq \gamma_{k}, \quad c_{1, k}, c_{2, k} \geq 1
$$

Proof. Since $\Psi_{k}^{-1}(s)=s^{\frac{1}{\gamma_{k}}}$, Hypothesis 2 hold for $k=1$. Based on the fact $\beta_{k} \leq \gamma_{k}$, Hypothesis 3 hold. Noting that

$$
\frac{s \phi_{k}^{\Delta}(s)}{\phi_{k}(s)}=\frac{s \Delta\left(s^{c_{1, k}}\right)}{s^{c_{1, k}}} \geq \frac{s c_{1, k} s^{c_{1, k}-1}}{s^{c_{1, k}}}=c_{1, k}, \quad \varphi_{k}(s)=s^{c_{2, k}} \geq s .
$$

Then Hypothesis 4 hold for $L=c_{1, k}$. Hypothesis 5 is also true due to $\int_{s}^{\infty} \phi_{k}(s) \Delta s=\infty$. 
We take $\delta(s)=s$ in (17), then

$$
\begin{gathered}
\int_{2}^{\infty} s e_{\frac{c_{1, k}-1}{t(t+1)}}\left(s, s_{1}\right) \Delta s=\int_{2}^{\infty} s \prod_{k=2}^{\infty}\left(1+\frac{c_{1, k}-1}{k(k+1)}\right) \Delta s \\
\geq \quad \int_{2}^{\infty} s\left(1+\sum_{k=2}^{\infty} \frac{c_{1, k}-1}{k(k+1)}\right) \Delta s=\int_{2}^{\infty} s\left(1+\frac{c_{1, k}-1}{2}\right) \Delta s=\infty .
\end{gathered}
$$

Based on Theorem 1, we know Equation (24) is oscillatory or tends to zero.

Example 2. Consider the equation on $\mathbb{T}=\mathbb{R}$,

$$
L_{n}(s)+\sum_{k=1}^{m} s^{c_{1, k}}\left(y\left(\tau_{k}(s)\right)\right)^{\mathcal{c}_{2, k}}=0, \quad s \in[2, \infty),
$$

where $\Psi_{k}(s)=s^{\gamma_{k}}, \lambda_{k}(s)=s^{\beta_{k}}$ and $\gamma_{k}$ is the quotient of odd positive integers satisfies

$$
\beta_{k} \leq \gamma_{k}(k=1,2, \cdots, n-1), \quad \beta_{n}-\min _{1 \leq k \leq m}\left\{c_{1, k}+1\right\} \leq \gamma_{n}, \quad \prod_{k=1}^{n} \gamma_{k} \leq 1, \quad c_{2, k} \geq 1, \quad c_{1, k} \leq-1 \text {. }
$$

Proof. Clearly, Hypotheses 1-4 hold same as Example 1. Using the fact that $\beta_{n}-\min _{1 \leq k \leq m}\left\{c_{1, k}+1\right\} \leq \gamma_{n}$, we have

$$
\begin{aligned}
& \int_{t}^{\infty} \Psi_{n}^{-1}\left(\frac{1}{\lambda_{n}(s)} \int_{s}^{\infty} \sum_{k=1}^{m} \phi_{k}(u) \Delta u\right) \Delta s \\
= & \int_{t}^{\infty}\left(\frac{1}{s^{\beta_{n}}} \sum_{k=1}^{m}\left(-\frac{1}{1+c_{1, k}} s^{c_{1, k}+1}\right)^{\frac{1}{\gamma_{k}}} \Delta s=\infty,\right.
\end{aligned}
$$

which means Hypothesis 5 holds. Moreover

$$
\frac{\varphi_{k}(s)}{\Psi_{n}\left(\Psi_{n-1}\left(\cdots\left(\Psi_{1}(s)\right)\right)\right)}=\frac{s^{c_{2, k}}}{{ }_{s} \prod_{k=1}^{n} \gamma_{k}} \geq 2^{1}=2=N_{1},
$$

and

$$
\frac{s\left(s^{\prod_{k=1}^{n} \gamma_{k}}\right)^{\prime}}{{ }_{S} \prod_{k=1}^{n} \gamma_{k}} \geq \prod_{k=1}^{n} \gamma_{k}=N_{2}
$$

showing Hypotheses 6 and 7 hold.

We take $\delta(s)=\frac{1}{s}$ in (18), then $-\frac{\delta^{\Delta}(s)}{\delta^{\sigma}(s)}=\frac{1}{s}$. Based on the recursion Formula (15), we can easily check that there exist $C_{1}, C_{2}, D_{1}, D_{2}>0$ such that $\eta_{1}(s) \leq C_{1} s^{D_{1}}$ and $\eta_{2}(s) \leq$ $C_{2} s^{D_{2}}$. Moreover, we have $\int_{s}^{\infty} \sum_{k=1}^{m} \phi_{k}(t) \Delta t<\infty$. Then there exist $m(s)=s^{E}$, where $E$ is sufficiently large, such that $B(s) \geq 1$. Therefore, we have

$$
\int_{2}^{\infty} B(s) e_{\frac{1}{t}}(s, 2) \Delta s=\int_{2}^{\infty} B(s) \frac{s}{2} \Delta s=\infty .
$$

Equation (25) is either oscillatory or tends to zero based on Theorem 2.

Example 3. Consider the equation on $\mathbb{T}=\mathbb{R}$,

$$
(y(s)+p(s) y(d(s)))^{(n)}+\sum_{k=1}^{m}\left(s^{c_{1, k}}\left(y\left(\tau_{k}(s)\right)\right)^{c_{2, k}}\right)=0, \quad s \in[1, \infty)
$$

where $c_{1, k}<-n$ and $c_{2, k}>0$ for all $k=1,2, \cdots, m$.

Proof. In this equation 


$$
\lambda_{k}(s) \equiv 1, \quad \Psi_{k}(s) \equiv s, \quad \Phi\left(s, y\left(\tau_{1}(s)\right), y\left(\tau_{2}(s)\right), \cdots, y\left(\tau_{m}(s)\right)\right)=\sum_{k=1}^{m} s^{c_{1, k}}\left(y\left(\tau_{k}(s)\right)\right)^{c_{2, k}}
$$

It is easy to check Hypotheses 1-4 and 9 hold. We can find that

$$
\begin{aligned}
& T^{*}\{\Phi(r)\}(s) \\
= & \int_{s}^{\infty} \Psi_{1}^{-1}\left(\frac { 1 } { \lambda _ { 1 } ( s _ { 1 } ) } \int _ { s _ { 1 } } ^ { \infty } \Psi _ { 2 } ^ { - 1 } \left(\frac{1}{\lambda_{2}\left(s_{2}\right)} \cdots\right.\right. \\
& \left.\left.\int_{s_{n-1}}^{\infty} \Psi_{n}^{-1}\left(\frac{1}{\lambda_{n}\left(s_{n}\right)} \int_{s_{n}}^{\infty} \sum_{k=1}^{m} s_{n+1}^{c_{1, k}} r^{c_{2, k}} \Delta s_{n+1}\right) \Delta s_{n} \cdots\right) \Delta s_{2}\right) \Delta s_{1} \\
= & \int_{s}^{\infty} \int_{s_{1}}^{\infty} \cdots \int_{s_{n-1}}^{\infty} \int_{s_{n}}^{\infty} \sum_{k=1}^{m} s_{n+1}^{c_{1, k}} r^{c_{2, k}} \Delta s_{n+1} \Delta s_{n} \cdots \Delta s_{2} \Delta s_{1} \\
= & \int_{s}^{\infty} \int_{s_{1}}^{\infty} \cdots \int_{s_{n-1}}^{\infty}-\sum_{k=1}^{m} \frac{r^{c_{2, k}}}{c_{1, k}+1} s_{n-1}^{c_{1, k}+1} \Delta s_{n} \cdots \Delta s_{2} \Delta s_{1} \\
= & \int_{s}^{\infty} \int_{s_{1}}^{\infty} \cdots \int_{s_{n-2}}^{\infty}(-1)^{2} \sum_{k=1}^{m} \frac{r^{c_{2, k}}}{\left(c_{1, k}+1\right)\left(c_{1, k}+2\right)} s_{n-1}^{c_{1, k}+2} \Delta s_{n-1} \cdots \Delta s_{2} \Delta s_{1} \\
= & \cdots \\
= & \int_{s}^{\infty}(-1)^{n} \sum_{k=1}^{m} \frac{r^{c_{2, k}}}{\prod_{j=1}^{n}\left(c_{1, k}+j\right)} s_{1}^{c_{1, k}+n} \Delta s_{1} \\
= & (-1)^{n+1} \sum_{k=1}^{m} \frac{r^{c_{2, k}}}{\prod_{j=1}^{n+1}\left(c_{1, k}+j\right)} s_{1}^{c_{1, k}+n+1}<\infty,
\end{aligned}
$$

where use the fact that $\lim _{t \rightarrow \infty} t^{c_{1, k}+l}=0$ for all $l=0,1,2, \cdots, n$. Based on Theorem 3, we know that Equation (26) has a nonoscillatory solution.

\section{Conclusions}

In this paper, we consider the higher order dynamic equations which have more universal functions $\Psi_{k}$ and more delay functions. Some properties of the equations are presented in lemmas. Then oscillatory and nonoscillatory criteria are established in theorems via Riccati technique and fixed point theorem. In the end, we provide some examples to check our results.

Author Contributions: Conceptualization, Y.-R.Z.; methodology, Y.-R.Z., Z.-X.M. and Y.-G.Z.; formal analysis, Y.-R.Z., Z.-X.M. and Y.-G.Z.; writing-original draft preparation, Z.-X.M. and X.-N.L.; writing-review and editing, Z.-X.M. and J.-F.T.; supervision, J.-F.T.; funding acquisition, J.-F.T. and Y.-R.Z. All authors have read and agreed to the published version of the manuscript.

Funding: This research was funded by the Fundamental Research Funds for the Central Universities under Grant 2015ZD29 and 2019MS117.

Institutional Review Board Statement: Not applicable.

Informed Consent Statement: Not applicable.

Data Availability Statement: Not applicable.

Acknowledgments: We thank Peng-Fei Sun and Tian-Chen Guo for their linguistic assistance during the preparation of this manuscript.

Conflicts of Interest: The authors declare no conflict of interest.

\section{References}

1. Hilger, S. Ein Maßkettenkalkül mit Anwendung auf Zentrumsmannigfaltigkeiten. Ph.D. Thesis, Universität Würzburg, Würzburg, Germany, 1988.

2. Bohner, M.; Georgiev, S.G. Multivariable Dynamic Calculus on Time Scales; Spriner: Cham, Switzerland, 2016.

3. Bohner, M.; Peterson, A. Dynamic Equations on Time Scales; Spriner: Boston, MA, USA, 2001.

4. Bohner, M.; Peterson, A. Advances in Dynamic Equations on Time Scales; Spriner: Boston, MA, USA, 2003. 
5. Martynyuk, A.A. Stability Theory for Dynamic Equations on Time Scales; Spriner: Boston, MA, USA, 2016.

6. Strogatz, S.H. Nonlinear Dynamics and Chaos: With Applications to Physics, Biology, Chemistry, and Engineering, 2nd ed.; CRC Press: Boca Raton, FL, USA, 2015.

7. Tarasov, V.E. Fractional Dynamics: Applications of Fractional Calculus to Dynamics of Particles, Fields and Media; Springer Science \& Business Media: Berlin/Heidelberg, Germany, 2011.

8. Magin, R.L. Fractional calculus models of complex dynamics in biological tissues. Comput. Math. Appl. 2010, 59, 1586-1593. [CrossRef]

9. Ma, Y.-T.; Li, W.-W. Application and research of fractional differential equations in dynamic analysis of supply chain financial chaotic system. Chaos Solitons Fractals 2020, 130, 10947. [CrossRef]

10. Erbe, L.; Peterson, A. Allan Riccati equations on a measure chain. Proc. Dyn. Syst. Appl. 2001, 3, 193-199.

11. Bohner, M.; Saker, S.H. Oscillation of second order nonlinear dynamic equations on time scales. Rocky Mt. J. Math. 2004, 34, 1239-1254. [CrossRef]

12. Hassan, T.S. Oscillation criteria for half-linear dynamic equations on time scales. J. Math. Anal. Appl. 2008, 345, 176-185. [CrossRef]

13. Erbe, L.; Hassan, T.S.; Peterson, A.; Saker, S.H. Oscillation criteria for half-linear delay dynamic equations on time scales. Nonlinear Dyn. Syst. Theory 2009, 9, 51-68.

14. Erbe, L.; Peterson, A.; Saker, S.H. Oscillation criteria for second-order nonlinear delay dynamic equations. J. Math. Anal. Appl. 2007, 333, 505-522. [CrossRef]

15. Agwo, H.A.; Khodier, A.M.M.; Hassan, H.A. Oscillation criteria of second order half linear delay dynamic equations on time scales. Acta Math. Appl. Sin. Engl. Ser. 2017, 33, 83-92. [CrossRef]

16. Deng, X.-H.; Wang, Q.-R.; Zhou, Z. Oscillation criteria for second order nonlinear delay dynamic equations on time scales. Appl. Math. Comput. 2015, 269, 834-840. [CrossRef]

17. Jia, B.-G.; Erbe, L.; Peterson, A. An oscillation theorem for second order superlinear dynamic equations on time scales. Appl. Math. Comput. 2013, 219, 10333-10342.

18. Zhu, Y.R.; Mao, Z.X.; Liu, S.P.; Tian, J.F. Oscillation criteria of second-order dynamic equations on time scales. Mathematics 2021, 9, 1867. [CrossRef]

19. Baculikova, B. Oscillation and asymptotic properties of second Order half-linear differential equations with mixed deviating arguments. Mathematics 2021, 9, 2552. [CrossRef]

20. Zhou, Y.; He, J.W.; Ahmad, B.; Alsaedi, A. Necessary and sufficient conditions for oscillation of fourth order dynamic equations on time scales. Adv. Differ. Equ. 2019, 2019, 308. [CrossRef]

21. Sui, Y.; Han, Z.-L. Oscillation of third-order nonlinear delay dynamic equation with damping term on time scales. J. Appl. Math. Comput. 2018, 58, 577-599. [CrossRef]

22. Zhou, Y. Nonoscillation of higher order neutral dynamic equations on time scales. Appl. Math. Lett. 2019, 94, 204-209. [CrossRef]

23. Anderson, D.R.; Zafer, A. Nonlinear oscillation of second-order dynamic equations on time scales. Appl. Math. Lett. 2009, 22, 1591-1597. [CrossRef]

24. Agarwal, R.P.; Bohner, M.; Li, T.; Zhang, C. Oscillation criteria for second-order dynamic equations on time scales. Appl. Math. Lett. 2014, 31, 34-40. [CrossRef]

25. Chatzarakis, G.E.; Grace, S.R.; Jadlovská, I. On the sharp oscillation criteria for half-linear second-order differential equations with several delay arguments. Appl. Math. Comput. 2021, 397, 125915. [CrossRef]

26. Sun, T.-X.; Yu, W.-Y.; Xi, H.-J. Oscillatory behavior and comparison for higher order nonlinear dynamic equations on time scales. J. Appl. Math. Inform. 2012, 30, 289-304.

27. Wu, X.; Sun, T.; Xi, H.; Chen, C. Kamenev-type oscillation criteria for higher-order nonlinear dynamic equations on time scales. Adv. Differ. Equ. 2013, 2013, 248. [CrossRef]

28. Sun, T.-X.; Yu, W.-Y.; He, Q.-L. New oscillation criteria for higher order delay dynamic equations on time scales. Adv. Differ. Equ. 2014, 2014, 328. [CrossRef]

29. Zhang, Z.; Dong, W.; Li, Q.; Liang, H. Positive solutions for higher order nonlinear neutral dynamic equations on time scales. Appl. Math. Model. 2009, 33, 2455-2463. [CrossRef]

30. Sun, T.; Xi, H.; Peng, X.; Yu, W. Nonoscillatory solutions for higher-order neutral dynamic equations on time scales. Abstr. Appl. Anal. 2010, 2010, 428963. [CrossRef]

31. Chen, D.-X. Oscillation and asymptotic behavior for nth-order nonlinear neutral delay dynamic equations on time scales. Acta. Appl. Math. 2010, 109, 703-719. [CrossRef]

32. Tao, C.-Y.; Sun, T.-X.; Xi, H.-J. Existence of the nonoscillatory solutions of higher order neutral dynamic equations on time scales Adv. Differ. Equ. 2015, 2015, 279. [CrossRef]

33. Qiu, Y.-C.; Wang, Q.-R. Existence of nonoscillatory dolutions to higher-order nonlinear neutral dynamic equations on time scales. Bull. Malays. Math. Sci. Soc. 2018, 41, 1935-1952. [CrossRef]

34. Chen, Y.-S. Existence of nonoscillatory solutions of nth order neutral delay differential equations. Funkc. Ekvacioj 1992, 35, 557-570. 